\title{
Table of Contents - Volume Two
}

Introduction to Leptocephali. By DAvID G. SMITH

Significance of the Leptocephalus Larva. By William H. Hulet

and C. RICHARD ROBINS

669

Orders Anguilliformes and Saccopharyngiformes: Leptocephali.

By DAVID G. SMITH

679

Family Synaphobranchidae: Leptocephali. By DAVID G. SMITH

Family Moringuidae: Leptocephali. By DAvID G. SMITH

699

Family Nettastomatidae: Leptocephali. By DAVID G. SMITH

704

Family Congridae: Leptocephali. By DAvID G. SMITH

723

Family Ophichthidae: Leptocephali. By MARK M. LEIBY

764

Family Anguillidae: Leptocephali. By DAVID G. SMITH

898

Family Muraenidae: Leptocephali. By DAVID G. SMITH

900

Family Derichthyidae: Leptocephali. By DAVID G. SMITH 917

Family Serrivomeridae: Leptocephali. By KENNETH A. TiGHE 921

Family Nemichthyidae: Leptocephali. By DAVID G. SMITH 925

Family Chlopsidae: Leptocephali. By DAvid G. SMITH 933

Family Heterenchelyidae: Leptocephali. By DAVID G. SMITH 943

Family Cyematidae: Leptocephali. By DAvID G. SMITH 944

Families Saccopharyngidae, Eurypharyngidae, and Monognathidae:

Leptocephali. By DAVID G. SMITH

Order Notacanthiformes: Leptocephali. By DAvID G. SMITH 955

Order Elopiformes: Families Elopidae, Megalopidae, and Albulidae:

Leptocephali. By David G. SMITH 961

Family Elopidae: Leptocephali $\quad 962$

Family Megalopidae: Leptocephali $\quad 968$

Family Albulidae: Leptocephali $\quad 970$

Unidentified Leptocephali. By David G. SMITH 973

Literature Cited $\quad 983$

INDEX 1019 
This page intentionally left blank 